Review

\title{
Deoxynivalenol-Induced Proinflammatory Gene Expression: Mechanisms and Pathological Sequelae
}

\author{
James J. Pestka ${ }^{1,2,3}$
}

1 Department of Microbiology and Molecular Genetics, Michigan State University, East Lansing, MI 48824, USA; E-Mail: Pestka@msu.edu; Tel.: +1-517-353-1709; Fax: +1-517-353-8963

2 Department of Food Science and Human Nutrition, Michigan State University, East Lansing, MI 48824, USA

3 Center for Integrative Toxicology, Michigan State University, East Lansing, MI 48824, USA

Received: 14 May 2010; in revised form: 25 May 2010 / Accepted: 28 May 2010 /

Published: 1 June 2010

\begin{abstract}
The trichothecene mycotoxin deoxynivalenol (DON) is commonly encountered in human cereal foods throughout the world as a result of infestation of grains in the field and in storage by the fungus Fusarium. Significant questions remain regarding the risks posed to humans from acute and chronic DON ingestion, and how to manage these risks without imperiling access to nutritionally important food commodities. Modulation of the innate immune system appears particularly critical to DON's toxic effects. Specifically, DON induces activation of mitogen-activated protein kinases (MAPKs) in macrophages and monocytes, which mediate robust induction of proinflammatory gene expressioneffects that can be recapitulated in intact animals. The initiating mechanisms for DON-induced ribotoxic stress response appear to involve the (1) activation of constitutive protein kinases on the damaged ribosome and (2) autophagy of the chaperone GRP78 with consequent activation of the ER stress response. Pathological sequelae resulting from chronic low dose exposure include anorexia, impaired weight gain, growth hormone dysregulation and aberrant $\operatorname{Ig} \mathrm{A}$ production whereas acute high dose exposure evokes gastroenteritis, emesis and a shock-like syndrome. Taken together, the capacity of DON to evoke ribotoxic stress in mononuclear phagocytes contributes significantly to its acute and chronic toxic effects in vivo. It is anticipated that these investigations will enable the identification of robust biomarkers of effect that will be applicable to epidemiological studies of the human health effects of this common mycotoxin.
\end{abstract}


Keywords: deoxynivalenol (DON); translation inhibition; macrophage; monocyte; cytokine; ribosome; ER stress

\section{Introduction}

Consumption of bread made from overwintered cereal grains infested with the mold Fusarium during World War II in the USSR resulted in massive outbreaks of alimentary toxic aleukia (ATA) [1]. ATA, a frequently fatal disease, involves both the immune and gastrointestinal systems and, as its name implies, evokes symptoms that included diarrhea, vomiting, leukopenia, hemorrhage and shock. Human gastroenteritis with nausea, diarrhea and vomiting as primary symptoms has been similarly linked to Fusarium-contaminated foods in Japan, Korea [2], China [3] and India [4]. Retrospective studies have demonstrated that a commonality among the fusaria isolated from such outbreaks is their capacity to produce a class of highly toxic secondary metabolites known as trichothecenes.

Trichothecene mycotoxins are low molecular weight $(\approx 200-500 \mathrm{D})$ sesquiterpenoids that contain both a common 9, 10 double bond and 12, 13 epoxide group as well as varied substituent groups that contribute significantly to their toxic potential. Of the over 200 members of this intriguing family that have been so far described [5-7], deoxynivalenol (DON) is very commonly encountered in cereal-based foods throughout the world [8]. High dose, acute exposure of sensitive animal species to DON, most notably pigs (e.g., 15-20 ppm in diet), elicits abdominal distress, increased salivation, malaise, diarrhea and emesis [9-14]. Contrastingly, prolonged low dose feeding of DON to experimental pigs and mice (e.g., 1-10 ppm) impairs weight gain, causes anorexia and possibly interferes with nutritional efficiency [13]. DON can stimulate or suppress immune function depending on dose, exposure frequency, timing and the functional immune assay being used [15]. As will be discussed below, this diverse spectrum of effects is likely to result from differences in intensity and duration of kinase signaling and the extent of resultant gene expression.

Large-scale epidemics of Fusarium infection in wheat, barley and corn with corresponding DON contamination are increasingly being observed in the U.S. and other parts of the world, possibly as inadvertent outcomes of expanded use of "no-till farming", inappropriate crop rotation and climate change. Recent biomarker studies indicate that most persons who consume wheat-containing foods are regularly exposed to DON [16,17]. Not surprisingly, there is growing global concern over the possibility of adverse human health outcomes resulting from acute and chronic DON consumption. Understanding the mode of action of natural toxins such as DON facilitates accurate prediction of potential toxic effects, thereby enabling more precise science-based risk assessment and risk management.

Studies in our laboratory and others have revealed that the innate immune system plays an intricate role in DON toxicity. The purpose of this review is to discuss (1) the molecular mechanisms that underlie DON toxicity with a specific focus on mononuclear phagocytes and (2) the relationship between DON-induced proinflammatory gene expression and downstream pathologic sequelae. 


\section{DON Targets Mononuclear Phagocytes}

\subsection{In vitro effects of $D O N$}

Primary cultures and cell lines derived from bone marrow, gut epithelium, liver, lymphoid tissue, kidney, lung and various cancers have been employed to assess DON's toxic effects. Leukocytes, most notably those of mononuclear phagocyte lineage, appear to be particularly responsive to DON [15]. Exposure of macrophages and monocytes to DON at low or moderate concentrations (i.e., partially inhibit translation) will selectively induce proinflammatory gene expression, but extended exposures to high concentrations (i.e., completely inhibit translation) can cause cell death. Susceptibility to apoptosis induction can vary greatly among cell types, with primary cells sometimes being more resistant [18].

\subsection{Mechanisms for DON inhibition of translation}

It has been proposed that trichothecenes including DON inhibit protein synthesis by binding to the peptidyl transferase region of the ribosome and interfering with initiation and elongation [19,20]. In addition to this canonical mechanism, DON might suppress protein synthesis in at least three other ways. For example, incubation of cloned macrophages with DON can result in lesions in the 28s rRNA which might render the 60s subunit non-functional [21]. In addition, DON induces activation of a ribosome-associated kinase known as double-stranded RNA-associated protein kinase (PKR) [22]. Upon activation, PKR can phosphorylate eukaryotic initiation factor $2 \alpha$ (EIF2 $\alpha$ ), thereby preventing translation [23]. Finally, it has recently been observed that DON can upregulate a large number of microRNAs (miRNAs) associated with selective gene downregulation [24]. Since many of these DON-induced miRNAs specifically correspond to ribosomal proteins, it is possible that those miRNA downregulate ribosome synthesis thus enabling DON-exposed cells to economize and redistribute resources needed for survival.

\subsection{Mechanisms for DON-induced proinflammatory gene upregulation}

Robust upregulation of specific cytokines, chemokines and other inflammation-related proteins by DON in vitro is preceded by elevations of their corresponding mRNAs [15] (Table 1). For example, DON induces mRNAs for TNF- $\alpha$ and IL-6 in macrophages [25,26], IL-8 in monocytes [27,28] and IL-2 expression in T cells $[29,30]$. The premise that DON and other translational inhibitors selectively drive overexpression of specific proteins appears at first to be intuitively contradictory. However, it is not unreasonable to predict that the rapid (e.g., 1 to 2 h) and marked (e.g., 10- to 1000-fold) upregulation of selected mRNAs in cells with partially suppressed translation would dramatically skew the expressed proteome. This concept is supported by comparable observations made for the ribotoxic proteins ricin and Shiga toxin [31-33].

Upregulation of mRNA expression by DON involves both transcriptional and post-transcriptional processes [15,34-36]. DON induces transcription factor expression (e.g., c-Fos, Fra-2, c-Jun , JunB, EGR1, ATF3) [37,38] and transcription factor activation (e.g., NF-кB, CREB, AP-1 and C/EBP) [29,39-42]. These transcription factors specifically regulate expression of inflammation- and 
immune-related genes. DON-induced transactivation has been confirmed using promoter-reporter studies assays $[28,34,43,44]$.

Table 1. Genes upregulated by deoxynivalenol in mice.

\begin{tabular}{l|l}
\hline \multicolumn{1}{c|}{ Gene Family } & \multicolumn{1}{c}{ Gene } \\
\hline Proinflammatory Cytokines & IL-1 $\alpha$, IL-1 $\beta$, IL-6, IL-11, TNF- $\alpha$, TGF- $\beta$ \\
T Cell Cytokines & IFN- $\gamma$, IL-2 \\
Chemokines & MIP-2, MCP-1, Crg-2, CINC-1, MCP-3 \\
Transcription Factors & cFos, cJun, Fra-2, Jun-B, NR4a1 \\
Phosphatases & MKP1, CNAb, Ptpn8, Ptprj \\
Suppressors of Cytokine Signaling (SOCS) & CIS1,SOCS1, SOCS2, SOCS3 \\
Other & Cox-2, C3aR \\
\hline
\end{tabular}

See references $[26,34,48,83,100,120]$.

DON also stabilizes mRNAs for TNF- $\alpha$, IL-6 and COX-2 [25,43,45], and IL-8 [39]. Enhanced mRNA stability relates to AUUUA motifs in the 3'-untranslated region (3'-UTR) of mRNAs that target transcripts for rapid degradation. Involvement of these 3' UTRs has been verified in studies of DON-induced COX-2 [43] and IL-8 mRNAs [39] stabilization. Notably, mRNA stability in the latter investigation was related to translocation of HuR/Elav-like RNA binding protein 1 (ELAVL1) from the nucleus to the cytosol and its association with 3'-untranslated region of the IL-8 transcript.

Transcriptional and post-transcriptional gene upregulation by DON is mediated by mitogen-activated protein kinases (MAPKs) known to be critical for signal transduction in the immune response. Activation of MAPKs by translational inhibitors was initially described in the landmark paper of Iordanov et al. [46] who termed it the "ribotoxic stress response". Three MAPK families are activated by DON in macrophage and monocyte cultures. These include: extracellular signal regulated protein kinase 1 and 2 (ERK1 and 2); (ii) p54 and p46 c-Jun N-terminal kinase 1 and 2 (JNK 1/2) and (iii) p38 [15].

While both ERK and p38 contribute to DON-induced transactivation of TNF- $\alpha$, IL- 6 and COX-2, only p38 is essential for trichothecene-mediated mRNA stabilization [25,34,43]. Analogous results have been observed for IL-8 in U937 human monocytes [47] and primary human mononuclear blood cell cultures [48]. Consistent with in vitro studies, DON sequentially induces (1) p38, ERK and JNK phosphorylation, (2) activation of the transcription factors AP-1, C/EBP, CREB and NF- $\kappa \mathrm{B}$ binding, and (3) proinflammatory cytokine mRNA expression in lymphoid tissues of the mouse [49].

When specific chemical inhibitors were used to screen for potential upstream kinases that could mediate DON-induced MAPK activation, both PKR and hematopoeitic cell kinase (Hck) were identified as candidate transducers $[22,50]$. PKR, a widely-expressed serine/threonine protein kinase, causes translational inhibition in an evolutionarily conserved antiviral response by phosphorylating [23]. PKR functions as a signal integrator for ligand-activated stress-activated protein kinase pathways leading to JNK and p38 activation as well as induction of TNF- $\alpha$, IL-6 and IL-12 expression. Hck, a member of the highly conserved Src-family of cytoplasmic protein tyrosine kinases, is specifically expressed in myelomonocytic cell lineages [51]. Hck transduces extracellular signals that regulate critical cellular processes such as proliferation, differentiation, migration and cytokine upregulation [52]. PKR and Hck are therefore likely to be essential for early steps in the ribotoxic stress response, 
and, furthermore, mediate initial events resulting in innate immune activation associated with macrophage exposure to DON (Figure 1).

Figure 1. DON-induced ribotoxic stress-Mechanism I. One mechanism for DON-induced ribotoxic stress is proposed to involve: (1) rapid DON uptake and binding to ribosome; (2) activation of ribosomal-associated PKR and Hck; (3) interaction of p38 with the ribosome; (4) p38 phosphorylation and (5) induction of proinflammatory genes.

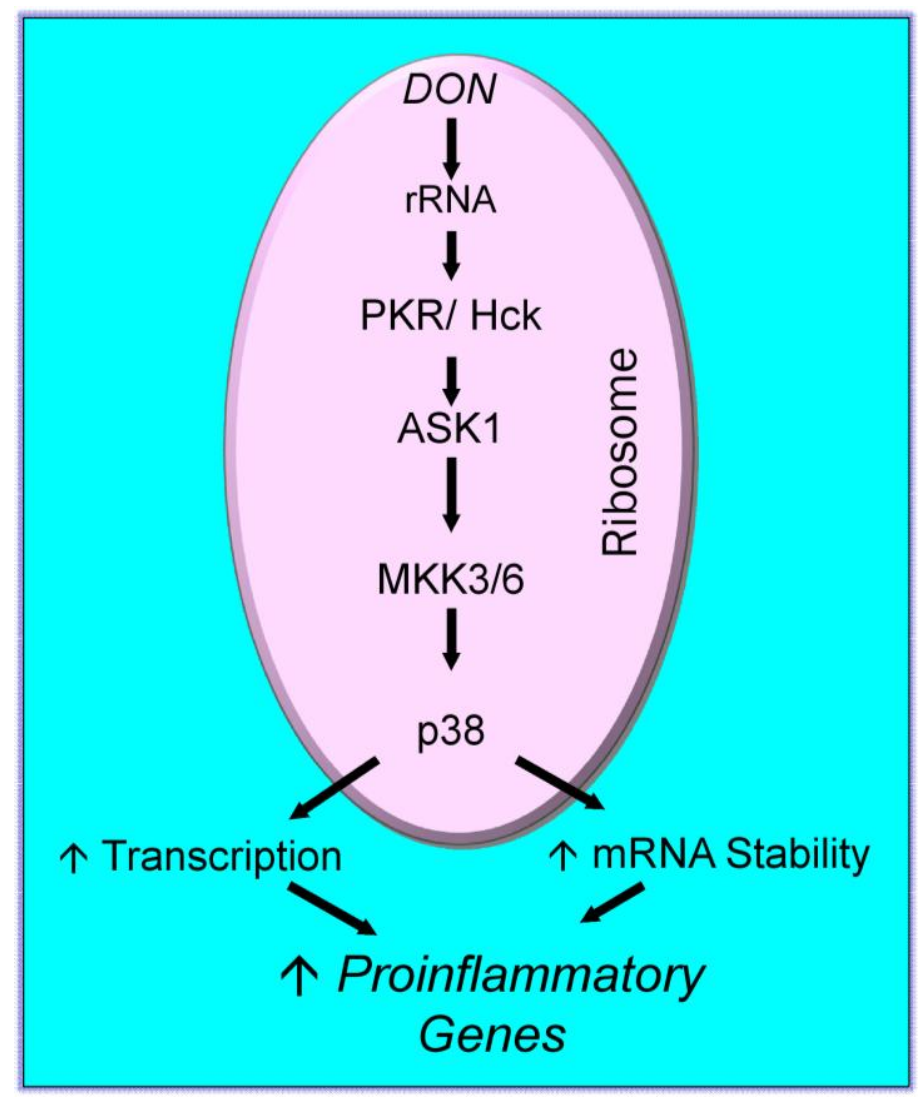

\subsection{Mechanisms for DON-induced cell death}

MAPK activation also precedes trichothecene-mediated apoptosis in RAW 264.7 macrophage and U937 monocyte models suggesting they have an equally important regulatory role in cell death [53]. When the contribution of p38 in mediating apoptosis and survival was investigated in DON-treated RAW 264.7 macrophages [54], it was observed that at concentrations that inhibit translation partially, DON induces p38 and ERK 1/2 phosphorylation within $15 \mathrm{~min}$ and this lasts up to $3 \mathrm{~h}$. DON-exposed cells exhibit increased caspase 3-dependent DNA fragmentation within $6 \mathrm{~h}$ that is suppressed and potentiated by p38 inhibition and ERK inhibition, respectively. DON evokes BAX translocation to mitochondria and cytochrome $\mathrm{C}$ release but does not affect mitochondrial membrane potential. In addition, DON also induces p38-dependent p53 activation. The p53 inhibitor PFT $\alpha$ and p53 siRNA knockdown suppress DON-induced caspase-3 activation and consequent DNA fragmentation. Accordingly, it has been proposed that DON induces competing apoptotic $(\mathrm{p} 38 \rightarrow \mathrm{p} 53 \rightarrow$ Bax $\rightarrow$ Mitochondria $\rightarrow$ Caspase-3) and survival (ERK $\rightarrow$ AKT/p90Rsk $\rightarrow$ Bad) pathways in RAW 264.7 macrophages. Since both PKR and Hck inhibition suppress DON-induced p53 activation, 
caspase-3 activity and apoptosis, these kinases are also likely to be upstream transducers in MAPK-regulated apoptosis [22,42].

On a cautionary note, while potent trichothecenes such as T-2 toxin cause robust cell death in lymphoid tissues and bone marrow, generally by apoptosis, DON's effects are more modest [20,53,55-61]. Exposure of mice to $25 \mathrm{mg} / \mathrm{kg}$ DON induces apoptosis in the thymus, bone marrow, spleen and Peyer's patches [62], whereas exposure to $12.5 \mathrm{mg} / \mathrm{kg}$ does not $[63,64]$ suggesting that relatively high DON dose (i.e., $1 / 2$ to $1 / 3 \mathrm{LD}_{50}$ ) is required for this programmed cell death. At 100 to $1000 \mathrm{ng} / \mathrm{mL}$, DON induces apoptosis in cloned macrophages and monocytes [22,54,65], T cells [66] and B cells [67]. Treatment with DON of thymus, spleen and bone marrow cultures at 250 to $500 \mathrm{ng} / \mathrm{mL}$ for $18 \mathrm{~h}$ induces apoptosis [68], but comparatively higher concentrations of DON (1-50 $\mu \mathrm{g} / \mathrm{mL})$ are required to cause modest apoptosis in T-cells, B-cells and IgA+ cells in mouse lymphoid cultures after $8 \mathrm{~h}$ [69]. Murine peritoneal macrophages are very resistant to DON even at concentrations up to $5 \mu \mathrm{g} / \mathrm{mL}$ for $12 \mathrm{~h}$ [70]. Comparable variability in DON-induced death induction among hematopoietic precursors has been observed [57,71]. Over all, these observations strongly suggest that inherent differences may exist in the balance between pro-apoptotic and anti-apoptotic pathways within individual phenotypes. Extrapolation of apoptosis studies conducted in cloned cells, often derived from tumors, to those carried out in primary cultures or in intact animals must be therefore performed with caution.

\section{Initiating Events in the Ribotoxic Stress Response}

Although there is recent evidence that DON interacts with both the 40S and 60S ribosomal units [72], the specific role of the ribosome in DON-induced MAPK activation and proinflammatory gene expression has not been fully delineated. Two possible contributing mechanisms identified to date are the direct activation of ribosome-associated kinases (Figure 1) and indirect activation via endoplasmic reticulum (ER) stress response (Figure 2).

It has been increasingly recognized that cells are able to sense damage-associated molecular patterns and evoke stress responses as a result [73]. We hypothesized that following DON-mediated perturbation or damage to rRNA, the ribosome acts as the initial staging site for MAPKs [74]. DON-treated U937 human monocytes and RAW 264.7 murine macrophages ribosomes were therefore subjected to sucrose density gradient fractionation and fractions immunoblotted for $\mathrm{p} 38$. Both total and phosphorylated p38 were found to increase in those fractions containing ribosomal subunits and monosomes. DON induced a similar segregation of JNK and ERK into the ribosomal fractions with concurrent phosphorylation. These data suggest that ribosome might serve as a scaffold in the ribotoxic stress response. It has now been demonstrated that PKR and Hck associate constitutively with ribosomal fractions where they potentially integrate the initial "sensing" of DON-induced ribosomal RNA damage and subsequently mediate intracellular kinase signaling [75] (Figure 1).

DON and other trichothecenes differ from ribosome-inactivating proteins (RIPs) such as ricin because, being small molecules, they do not possess the inherent enzyme capacity to promote $28 \mathrm{~S}$ rRNA cleavage at the alpha-sarcin/ricin (S/R)-loop (A4256) under cell-free conditions [76]. However, incubation of RAW 264.7 cells with either DON or ricin promotes the cleavage of 28S rRNA at two other sites (A3560 and A4045) in the peptidyl transferase center. Furthermore, both DON and ricin induce RNase activity and RNase L mRNA expression. 
Figure 2. DON-induced ribotoxic stress-Mechanism II. The toxin increases unfolded protein concentration resulting in sequestering and degradation of GRP78 thereby evoking an ER stress. The resultant response includes upregulation of ATF6 and XBP1 resulting in CREB activation. Another hypothetical effect is activation of ASK1-mediated p38 activation (pink shade). Both actions could contribute to proinflammatory gene expression.

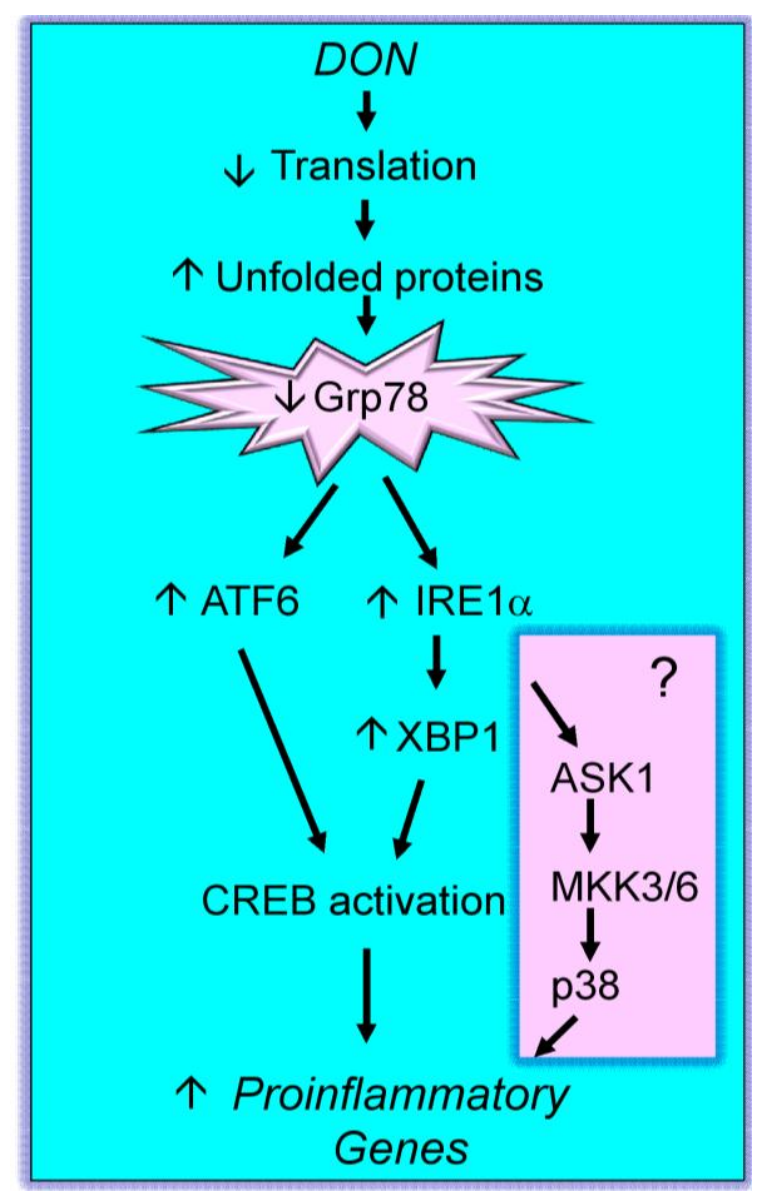

Thus, DON can promote intracellular 28S rRNA cleavage, potentially by facilitating the action of endogenous RNases and/or by upregulating RNase expression. Although it remains to be resolved whether these cleavages are upstream or downstream to the actual ribotoxic stress response, the findings support the possibility that DON interacts with the peptidyl transferase region of the $28 \mathrm{~S}$ rRNA in a similar fashion to ricin. It is tempting to speculate that DON-induced perturbation or damage at this site initiates kinase activation and recruitment. PKR is an attractive candidate for the sensing role since it has a dsRNA binding site that could bind to damaged rRNA.

DON interaction with the ribosome also results in an ER stress response (Figure 2). In support of this contention, DON-treated macrophages markedly decrease expression of cytoplasmic glucose regulated protein (GRP) 78, a chaperone known to mediate ER stress in peritoneal macrophages [18]. Since GRP78 mRNA is unchanged following DON treatment, GRP78 loss likely results from degradation rather than reduced expression. The ubiquitin-proteasome and autophagy-lysosomal pathways constitute two major avenues for protein degradation [77]. DON-induced GRP78 degradation is cathepsin- and calpain-dependent but not proteosome-dependent suggesting an autophagy pathway is involved [18]. Potential mechanisms for DON-induced GRP78 autophagy are not known. Ribosome disruption and/or translation arrest might cause accumulation of misfolded 
proteins that are sequestered by GRP78. Resultant GRP-containing complexes could enter into the autophagy pathway and be degraded. It should be noted that while the autophagy-lysosomal predominates here, DON has been demonstrated to upregulate genes associated with regulation and structure of the proteosome complex [78] as well as proteins of the ubiquitin-proteosome complex [79].

GRP78 regulates two transcription factors, X-box binding protein 1 (XBP1) and activating transcription factor 6 (ATF6). These bind to cAMP-response element (CRE) and drive expression of CRE-dependent genes. DON treatment increases levels of ATF6 as well as IRE1 $\alpha$ protein and its modified products spliced XBP1 mRNA and XBP1 protein [18]. ATF6 but not XBP1 knockdown partially inhibits DON-induced IL-6 expression.

It is also possible that DON-induced ER stress contributes to MAPK activation. Specifically, it is known that (1) IRE 1 activation mediates ASK1 phosphorylation and (2) DON induces ASK1 phosphorylation [75]. Accordingly, the ER stress response could thus be a second complementary pathway by which DON and other trichothecenes affect innate immune function (Figure 2). Three other translation inhibitors, T-2 toxin, Shiga toxin and ricin, also induce GRP78 degradation, further suggesting this pathway could be a common mechanism for ribotoxic stress.

It should be further noted that while ER stress initially triggers evolutionarily conserved signal-transduction events designed to ameliorate unfolded protein accumulation in the ER, if these events are severe or protracted, they can induce apoptosis. In the future, it will be desirable to identify critical events upstream and downstream of GRP degradation as well as ascertain the comparative contributions of this pathway to upregulation of inflammatory genes and apoptosis.

While two possible mechanisms of ribotoxic stress have been proposed here for DON, it does not preclude the existence of alternative mechanisms for this or other agents. For example palytoxin does not inhibit translating ribosomes under cell-free conditions but requires translating ribosomes to transduce signals that activate JNKI [81]. Osman et al. [79] observed in a proteomic analysis of EL-4 thymoma cells that DON induced expression of My-binding protein A (MYBBP1A). This latter protein interacts with many ribosomal proteins and can interact with a number of transcription factors [82].

\section{Pathological Sequelae to DON-Induced Innate Immune Activation}

What is the in vivo relevance of the DON-induced proinflammatory gene expression? DON is rapidly distributed throughout the body following oral exposure of mice $[83,84]$ and thus would be present in many tissues containing mononuclear phagocytes. Consistent with in vitro studies, DON concomitantly induces a wide array of proinflammatory cytokines and chemokines that are detectable in spleen, liver, kidney and lung [37,49,84-89]. Ribotoxic stress in mononuclear phagocytes, with consequent induction of proinflammatory gene expression, is likely to be critical for the induction of acute and chronic sequelae associated with DON poisoning in experimental animals (Figure 3).

Aberrant elevation of inflammatory mediators, often referred to as a cytokine storm [90], mediate the shock-like effects of lipopolysaccharide (LPS) [91] and might likewise contribute to acute toxic effects of DON. Indeed, LPS and other toll-like receptor (TLR) agonists potentiate DON toxicity in mice $[62,89,92-95]$. Interestingly, DON has been reported to damage the integrity of intestinal cells and allow increased bacterial translocation [96-99]. Such collateral damage could greatly magnify DON toxicity. 
Figure 3. Potential downstream pathological sequelae associated with DON-induced ribotoxic stress.

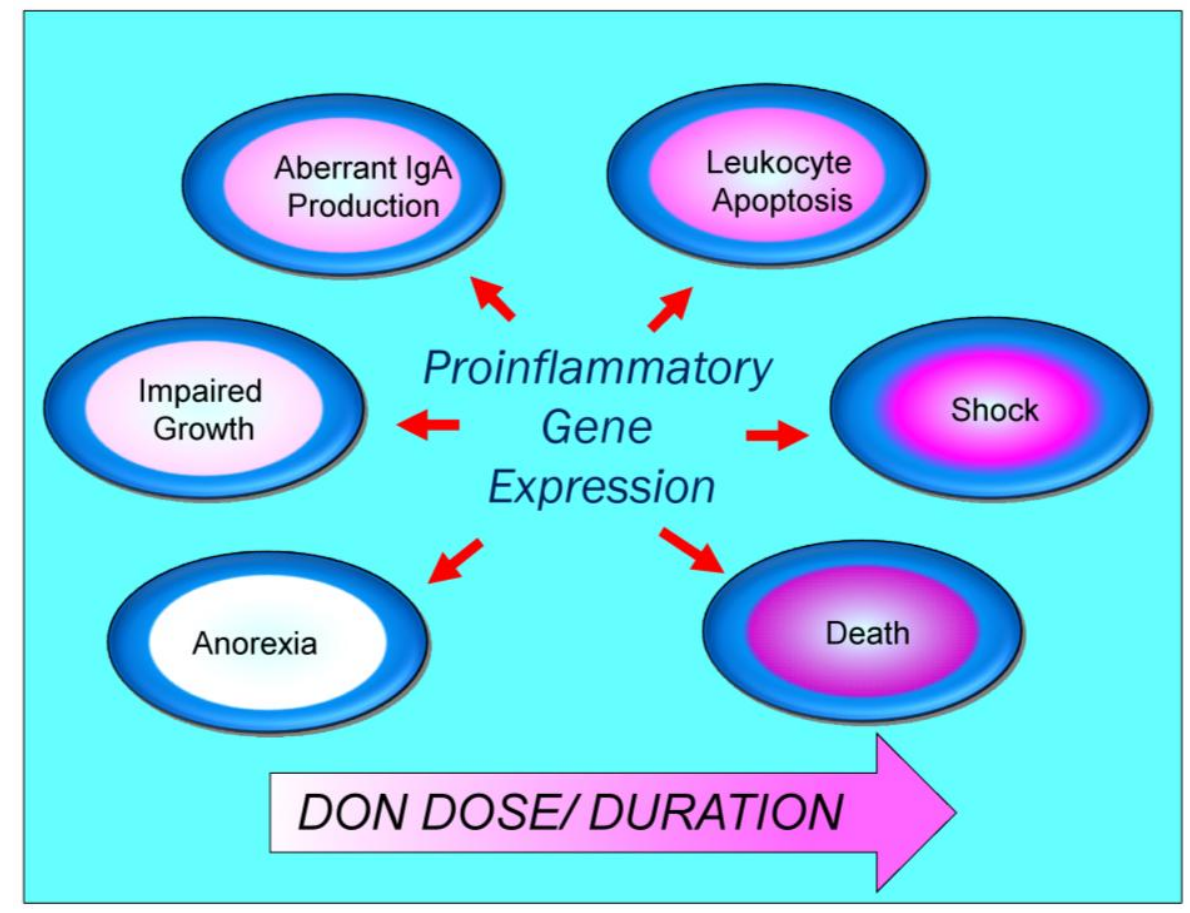

Proinflammatory cytokines induce several suppressors of cytokine signaling (SOCS). DON upregulates mRNA expression of four well-characterized SOCS (CIS [cytokine-inducible SH2 domain protein], SOCS1, SOCS2, and SOCS3) following cytokine upregulation [100]. DON specifically induces SOCS3 mRNAs in muscle, spleen and liver, in addition to CIS1, SOCS1, and SOCS2 in other tissues. Notably, hepatic SOCS3 mRNA and protein are sensitive indicators of DON exposure. SOCS induction could be an essential feedback mechanism for downscaling proinflammatory gene effects and their subsequent pathological sequelae. Another complementary feedback mechanism reported for DON is the upregulation of mitogen-activated protein kinase phosphatase 1 [86].

Induction of proinflammatory cytokines could contribute DON impairment of appetite and weight gain observed by either directly affecting the brain and nervous system [101] and/or SOCS-mediated deregulation of growth hormone $(\mathrm{GH})$ signaling [102]. In support of the latter, subchronic dietary exposure of young mice to DON decreases hepatic insulin-like growth factor acid labile subunit (IGFALS) mRNA expression and downregulates plasma insulin-like growth factor 1 (IGF1) and IGFALS levels, as well as attenuates weight gain [103]. Liver IGFALS mRNA levels decrease within $2 \mathrm{~h}$ of oral exposure to DON (0.5 to12.5 mg/kg body weight), while $0.1 \mathrm{mg} / \mathrm{kg}$ body weight DON is without effect. The latter effects co-occur with robust hepatic SOCS3 upregulation with and without exogenous GH treatment. Oral DON exposure in the mouse therefore appears to dysregulate the GH axis by impairing two critical growth-related proteins, IGFALS and IGF1. While their specific contributions to DON-induced food intake and growth retardation still remain to be elucidated, both proteins might be useful as serum biomarkers for DON effect [104].

Prolonged DON feeding to mice causes dramatic elevations in total serum IgA and serum IgA-immune complexes (IgA-IC) and polymeric IgA [105,101]. DON-exposed mice further exhibit kidney mesangial IgA accumulation, electron dense mesangial deposits and hematuria [106] —all hallmarks of 
human IgA nephropathy, the most common type of glomerulonephritis worldwide [107]. Following removal of DON from mouse diet, toxin-induced elevations in serum $\operatorname{Ig} \mathrm{A}, \operatorname{Ig} \mathrm{A}-\mathrm{IC}$, mesangial $\operatorname{Ig} \mathrm{A}$ and hematuria persist for several months [108]. Peyer's patches (PP) from DON-exposed mice contain increased numbers of membrane IgA-bearing cells [109]. PP lymphocytes from PP and spleens of DON-fed mice produce significantly more IgA than control cultures prepared from mice fed clean diet [110]. Accordingly, in mice exposed to DON, there is rapid polyclonal activation of IgA-secreting plasma cells in the gut at the PP level and this is similarly reflected in the systemic compartment.

DON does not exert adjuvant effects when orally administered with exogenous mucosal antigens, but rather, polyclonally induces production of IgAs that are reactive with a variety of intestinal and self antigens [111-114]. Polyspecific, autoreactive IgA may contribute to kidney immune complex deposition or direct binding to the kidney mesangium. IL-6, which is robustly induced upon DON exposure in vivo and ex vivo [87,115] is known to drive differentiation of B cells to IgA production [116]. The macrophages appear to contribute to IgA production and IgA nephropathy in DON-exposed mice by upregulating IL-6 [117]. Serum IgA, IgA immune complexes, kidney mesangial IgA and hematuria were significantly higher in DON-fed wild-type mice than toxin-fed IL-6 KO [118] suggesting that IL-6 is a requisite cytokine for DON-induced IgA production and resultant IgAN.

Taken together, DON-induced proinflammatory cytokine production by mononuclear phagocytes in vivo is likely to contribute to many pathological sequelae associated with exposure to trichothecene.

\section{Conclusions}

Substantive questions remain regarding the risks posed to humans from acute and chronic DON ingestion and how to manage these risks without threatening food security. As demonstrated here, DON dramatically induces MAPK activation in vitro and in vivo in macrophages and monocytes which in turn, mediate robust induction of both proinflammatory gene expression and, at very high doses, apoptosis. Mechanisms for the DON-induced ribotoxic stress response appear to involve the (1) activation of constitutive ribosomal kinases and mobilization of MAPKs to the damaged ribosome (Figure 1) and (2) autophagy of GRP78 with consequent action of an ER stress response (Figure 2). Downstream pathophysiologic sequelae of DON-induced ribotoxic stress in mononuclear phagocytes include chronic toxic effects such as anorexia, growth and aberrant IgA production as well as acute toxicity at high doses. It is anticipated that current and future investigations will identify robust biomarkers of effect that when coupled with biomarkers of exposure [119] will facilitate epidemiological studies of potential DON-associated human illnesses.

\section{Acknowledgements}

This project was funded in part by Public Health Service Grant ES 3358 from the National Institute for Environmental Health Sciences. This work was also supported in part by the USDA, under a cooperative project with U.S. Wheat and Barley Scab Initiative. Any findings, opinions, conclusions or recommendations expressed in this publication are those of the author and do not necessarily reflect the views of USDA. The author acknowledges technical assistance from Mary Rosner. 


\section{References}

1. Joffe, A.Z. Fusarium poae and F. sporotrichoides as principal causal agents of alimentary toxic aleukia. In Mycotoxic Fungi, Mycotoxins,Mycotoxicoses: An Encyclopedic Handbook; Wyllie, T.D., Morehouse, L.G., Eds.; Dekker: New York, NY, USA, 1978; pp. 21-86.

2. Yoshizawa, T. Trichothecenes: Chemical, Biological, and Toxicological Aspects. In Developments in Food Science; Ueno, Y., Ed.; Kodansha Ltd.: Tokyo, Japan, 1983; pp. 195-209.

3. Luo, X. Food Poisoning Caused by Fusarium Toxins; ILSI: Bangkok, Thailand, 1994; pp. 129-136.

4. Bhat, R.V.; Beedu, S.R.; Ramakrishna, Y.; Munshi, K.L. Outbreak of trichothecene mycotoxicosis associated with consumption of mould-damaged wheat production in Kashmir Valley, India. Lancet 1989, 1, 35-37.

5. Grove, J.F. Non-macrocyclic trichothecenes. Nat. Prod. Rep. 1988, 5, 187-209.

6. Grove, J.F. Macrocyclic trichothecenes. Nat. Prod. Rep. 1993, 10, 429-448.

7. Grove, J.F. Non-macrocyclic trichothecenes. Part 2. Prog. Chem. Org. Nat. Prod. 2000, 69, 1-70.

8. Canady, R.A.; Coker, R.D.; Rgan, S.K.; Krska, R.; Kuiper-Goodman, T.; Olsen, M.; Pestka, J.J.; Resnik, S.; Schlatter, J. Deoxynivalenol. Safety evaluation of certain mycotoxins in food. Fiftysixth report of the Joint FAO/WHO Expert Committee on Food Additives. International Programme on Chemical Safety, World Health Organization: Geneva, Switzerland, 2001; pp. 420-555.

9. Forsyth, D.M.; Yoshizawa, T.; Morooka, N.; Tuite, J. Emetic and refusal activity of deoxynivalenol to swine. Appl. Environ. Microbiol. 1977, 34, 547-552.

10. Friend, D.W.; Trenholm, H.L.; Elliot, J.I.; Thompson, B.K.; Hartin, K.E. Effect of feeding vomitoxin-contaminated wheat to pigs. Can. J. Anim. Sci. 1982, 62, 1211-1222.

11. Pestka, J.J.; Lin, W.S.; Miller, E.R. Emetic activity of the trichothecene 15-acetyldeoxynivalenol in swine. Food Chem. Toxicol. 1987, 25, 855-858.

12. Prelusky, D.B.; Trenholm, H.L. The efficacy of various classes of anti-emetics in preventing deoxynivalenol-induced vomiting in swine. Nat. Toxins 1993, 1, 296-302.

13. Rotter, B.A.; Prelusky, D.B.; Pestka, J.J. Toxicology of deoxynivalenol (vomitoxin). J. Toxicol. Environ. Health 1996, 48, 1-34.

14. Young, L.G.; McGirr, L.; Valli, V.E.; Lumsden, J.H.; Lun, A. Vomitoxin in corn fed to young pigs. J. Anim. Sci. 1983, 57, 655-664.

15. Pestka, J.J. Mechanisms of deoxynivalenol-induced gene expression and apoptosis. Food Addit. Contam. 2008, 25, 1128-1140

16. Turner, P.C.; Rothwell, J.A.; White, K.L. M.; Gong, Y.; Cade, J.E.; Wild, C.P. Urinary deoxynivalenol is correlated with cereal intake in individuals from the United Kingdom. Environ. Health Perspec. 2008, 116, 21-25.

17. Turner, P.C.; Taylor, E.F.; White, K.L. M.; Cade, J.E.; Wild, C.P. A comparison of 24 h urinary deoxynivalenol with recent v. average cereal consumption for UK adults. British J. Nutri. 2009, 102, 1276-1284. 
18. Shi, Y.H.; Porter, K.; Parameswaran, N.; Bae, H.K.; Pestka, J.J. Role of GRP78/BiP Degradation and ER Stress in Deoxynivalenol-Induced Interleukin-6 Upregulation in the Macrophage (vol 109, pg 247, 2009). Toxicol. Sci. 2009, 110, 249-250.

19. Ueno, Y. Trichothecenes: Chemical, Biological, and Toxicological Aspects. In Trichothecenes; Ueno, Y., Ed.; Elsevier Press: Amsterdam, The Netherlands, 1983; pp. 135-146.

20. Shifrin, V.I.; Anderson, P. Trichothecene mycotoxins trigger a ribotoxic stress response that activates c-Jun N-terminal kinase and p38 mitogen-activated protein kinase and induces apoptosis. J. Biol. Chem. 1999, 274, 13985-13992.

21. Li, M.; Pestka, J.J. Comparative induction of $28 \mathrm{~S}$ ribosomal RNA cleavage by ricin and the trichothecenes deoxynivalenol and T-2 toxin in the macrophage. Toxicol. Sci. 2008, 105, 67-78.

22. Zhou, H.R.; Lau, A.S.; Pestka, J.J. Role of double-stranded RNA-activated protein kinase R (PKR) in deoxynivalenol-induced ribotoxic stress response. Toxicol. Sci. 2003, 74, 335-344.

23. Williams, B.R. Signal integration via PKR. Sci. STKE 2001, 89, re2.

24. He, K.; Vines, L.; Pestka, J.J. Deoxynivalenol-induced modulation of microRNA expression in RAW 264.7 macrophages-A potential novel mechanism for translational inhibition. Toxicologist (Toxicol. Sci. Suppl.) 2010, 114, 310.

25. Chung, Y.J.; Zhou, H.R.; Pestka, J.J. Transcriptional and posttranscriptional roles for p38 mitogen-activated protein kinase in upregulation of TNF-alpha expression by deoxynivalenol (vomitoxin). Toxicol. Appl. Pharmacol. 2003, 193, 188-201.

26. Jia, Q.; Zhou, H.R.; Bennink, M.; Pestka, J.J. Docosahexaenoic acid attenuates mycotoxininduced immunoglobulin a nephropathy, interleukin-6 transcription, and mitogen-activated protein kinase phosphorylation in mice. J. Nutr. 2004, 134, 3343-3349.

27. Gray, J.S.; Bae, H.K.; Li, J.C.B.; Lau, A.S.; Pestka, J.J. Double-stranded RNA-activated protein kinase (PKR) mediates induction of IL-8 expression by deoxynivalenol, Shiga toxin 1 and ricin in monocytes. Toxicol. Sci. 2008. 105, 322-330.

28. Gray, J.S.; Pestka, J.J. Transcriptional regulation of deoxynivalenol-induced IL-8 expression in human monocytes. Toxicol. Sci. 2007, 99, 502-511.

29. Li, S.; Ouyang, Y.; Yang, G.H.; Pestka, J.J. Modulation of transcription factor AP-1 activity in murine EL-4 thymoma cells by vomitoxin (deoxynivalenol). Toxicol. Appl. Pharmacol. 2000, $163,17-25$.

30. Li, S.G.; Ouyang, Y.L.; Dong, W.M.; Pestka, J.J. Superinduction of IL-2 gene expression by vomitoxin (deoxynivalenol) involves increased mRNA stability. Toxicol. Appl. Pharmacol. 1997, 147, 331-342.

31. Korcheva, V.; Wong, J.; Lindauer, M.; Jacoby, D.B.; Iordanov, M.S.; Magun, B. Role of apoptotic signaling pathways in regulation of inflammatory responses to ricin in primary murine macrophages. Mol. Immunol. 2007, 44, 2761-2771.

32. Cherla, R.P.; Lee, S.Y.; Mees, P.L.; Tesh, V.L. Shiga toxin 1-induced cytokine production is mediated by MAP kinase pathways and translation initiation factor eIF4E in the macrophage-like THP-1 cell line. J. Leukoc. Biol. 2006, 79, 397-407.

33. Leyva-Illades, D.; Cherla, R.P.; Galindo, C.L.; Chopra, A.K.; Tesh, V.L. Global transcriptional response of macrophage-like THP-1 cells to Shiga toxin type 1. Infec. Immunity 2010, 78, 2454-2465. 
34. Moon, Y.; Pestka, J.J. Vomitoxin-induced cyclooxygenase-2 gene expression in macrophages mediated by activation of ERK and p38 but not JNK mitogen-activated protein kinases. Toxicol. Sci. 2002, 69, 373-382.

35. Moon, Y.; Pestka, J.J. Deoxynivalenol-induced mitogen-activated protein kinase phosphorylation and IL-6 expression in mice suppressed by fish oil. J. Nutr. Biochem. 2003, 14, 717-726.

36. Moon, Y.; Pestka, J.J. Cyclooxygenase-2 mediates interleukin-6 upregulation by vomitoxin (deoxynivalenol) in vitro and in vivo. Toxicol. Appl. Pharmacol. 2003, 187, 80-88.

37. Kinser, S.; Li, M.; Jia, Q.; Pestka, J.J. Truncated deoxynivalenol-induced splenic immediate early gene response in mice consuming (n-3) polyunsaturated fatty acids. J. Nutr. Biochem. 2005, $16,88-95$.

38. Nielsen, C.; Lippke, H.; Didier, A.; Dietrich, R.; Martlbauer, E. Potential of deoxynivalenol to induce transcription factors in human hepatoma cells. Molec. Nutri. Food Res. 2009, 53, 479-491.

39. Choi, H.J.; Yang, H.; Park, S.H.; Moon, Y. HuR/ELAVL1 RNA binding protein modulates interleukin-8 induction by muco-active ribotoxin deoxynivalenol. Toxicol. Appl. Pharmacol. 2009, 240, 46-54.

40. Ouyang, Y.L.; Li, S.; Pestka, J.J. Effects of vomitoxin (deoxynivalenol) on transcription factor NF-kappa B/Rel binding activity in murine EL-4 thymoma and primary CD4+ T cells. Toxicol. Appl. Pharmacol. 1996, 140, 328-336.

41. Wong, S.S.; Zhou, H.R.; Pestka, J.J. Effects of vomitoxin (deoxynivalenol) on the binding of transcription factors AP-1, NF-kappaB, and NF-IL6 in raw 264.7 macrophage cells. J. Toxicol. Environ. Health A 2002, 65, 1161-1180.

42. Zhou, H.R.; Jia, Q.; Pestka, J.J. Ribotoxic stress response to the trichothecene deoxynivalenol in the macrophage involves the SRC family kinase Hck. Toxicol. Sci. 2005, 85, 916-926.

43. Moon, Y.; Uzarski, R.; Pestka, J.J. Relationship of trichothecene structure to COX-2 induction in the macrophage: selective action of type B (8-keto) trichothecenes. J. Toxicol. Environ. Health A 2003, 66, 1967-1983.

44. Wong, S.S.; Schwartz, R.C.; Pestka, J.J. Superinduction of TNF-alpha and IL-6 in macrophages by vomitoxin (deoxynivalenol) modulated by mRNA stabilization. Toxicology 2001, 161, 139-149.

45. Jia, Q.; Zhou, H.R.; Shi, Y.; Pestka, J.J. Docosahexaenoic acid consumption inhibits deoxynivalenol-induced CREB/ATF1 activation and IL-6 gene transcription in mouse macrophages. J. Nutr. 2006, 136, 366-372.

46. Iordanov, M.S.; Pribnow, D.; Magun, J.L.; Dinh, T.H.; Pearson, J.A.; Chen, S.L.; Magun, B.E. Ribotoxic stress response: activation of the stress-activated protein kinase JNK1 by inhibitors of the peptidyl transferase reaction and by sequence-specific RNA damage to the alpha-sarcin/ricin loop in the 28S rRNA. Mol. Cell Biol. 1997, 17, 3373-3381.

47. Gray, J.S.; Bae, H.K.; Li, J.C.; Lau, A.S.; Pestka, J.J. Double-stranded RNA-activated protein kinase mediates induction of interleukin-8 expression by deoxynivalenol, Shiga toxin 1, and ricin in monocytes. Toxicol. Sci. 2008, 105, 322-330.

48. Islam, Z.; Gray, J.S.; Pestka, J.J. p38 Mitogen-activated protein kinase mediates IL-8 induction by the ribotoxin deoxynivalenol in human monocytes. Toxicol. Appl. Pharmacol. 2006, 213, 235-244. 
49. Zhou, H.R.; Islam, Z.; Pestka, J.J. Rapid, sequential activation of mitogen-activated protein kinases and transcription factors precedes proinflammatory cytokine mRNA expression in spleens of mice exposed to the trichothecene vomitoxin. Toxicol. Sci. 2003, 72, 130-142.

50. Zhou, H.R.;Jia, Q.;Pestka,J.J. Ribotoxic stress response to the trichothecene deoxynivalenol in the macrophage involves the SRC family kinase Hck. Toxicol. Sci. 2005, 85, 916-926.

51. Tsygankov, A.Y.; Shore, S.K. Src: regulation, role in human carcinogenesis and pharmacological inhibitors. Curr. Pharm. Des. 2004, 10, 1745-1756.

52. English, B.K.; Ihle, J.N.; Myracle, A.; Yi, T. Hck tyrosine kinase activity modulates tumor necrosis factor production by murine macrophages. J. Exp. Med. 1993, 178, 1017-1022.

53. Yang, G.H.; Jarvis, B.B.; Chung, Y.J.; Pestka, J.J. Apoptosis induction by the satratoxins and other trichothecene mycotoxins: relationship to ERK, p38 MAPK, and SAPK/JNK activation. Toxicol. Appl. Pharmacol. 2000, 164, 149-160.

54. Zhou, H.R.; Islam, Z.; Pestka, J.J. Induction of competing apoptotic and survival signaling pathways in the macrophage by the ribotoxic trichothecene deoxynivalenol. Toxicol. Sci. 2005, 87, 113-122.

55. Islam, Z.; Nagase, M.; Ota, A.; Ueda, S.; Yoshizawa, T.; Sakato, N. Structure-function relationship of T-2 toxin and its metabolites in inducing thymic apoptosis in vivo in mice. Biosci. Biotechnol. Biochem. 1998, 62, 1492-1497.

56. Islam, Z.; Nagase, M.; Yoshizawa, T.; Yamauchi, K.E.; Sakato, N. T-2 toxin induces thymic apoptosis in vivo in mice. Toxicol. Appl. Pharmacol. 1998, 148, 205-214.

57. Le Drean, G.; Auffret, M.; Batina, P.; Arnold, F.; Sibiril, Y.; Arzur, D.; Parent-Massin, D. Myelotoxicity of trichothecenes and apoptosis: An in vitro study on human cord blood CD34(+) hematopoietic progenitor. Toxicol. Vitro 2005, 19, 1015-1024.

58. Miura, K.; Nakajima, Y.; Yamanaka, N.; Terao, K.; Shibato, T.; Ishino, S. Induction of apoptosis with fusarenon-X in mouse thymocytes. Toxicology 1998, 127, 195-206.

59. Nagase, M.; Alam, M.M.; Tsushima, A.; Yoshizawa, T.; Sakato, N. Apoptosis induction by T-2 toxin: Activation of caspase-9, caspase-3, and DFF-40/CAD through cytosolic release of cytochrome c in HL-60 cells. Biosci. Biotechnol. Biochem. 2001, 65, 1741-1747.

60. Poapolathep, A.; Kumagai, S.; Suzuki, H.; Doi, K. Development of early apoptosis and changes in T-cell subsets in mouse thymocyte primary cultures treated with nivalenol. Exp. Mol. Pathol. 2004, 77, 149-152.

61. Yoshino, N.; Takizawa, M.; Akiba, H.; Okumura, H.; Tashiro, F.; Honda, M.; Ueno, Y. Transient elevation of intracellular calcium ion levels as an early event in T-2 toxin-induced apoptosis in human promyelotic cell line HL-60. Nat. Toxins 1996, 4, 234-241.

62. Zhou, H.R.; Harkema, J.R.; Hotchkiss, J.A.; Yan, D.; Roth, R.A.; Pestka, J.J. Lipopolysaccharide and the trichothecene vomitoxin (deoxynivalenol) synergistically induce apoptosis in murine lymphoid organs. Toxicol. Sci. 2000, 53, 253-263.

63. Islam, Z.; Pestka, J.J. Role of IL-1 beta in LPS potentiation of deoxynivalenol-induced leukocyte apoptosis in mice. Toxicol. Sci. 2003, 72, 1605.

64. Islam, Z.; Pestka, J.J. LPS priming potentiates and prolongs proinflammatory cytokine response to the trichothecene deoxynivalenol in the mouse. Toxicol. Appl. Pharmacol. 2006, 211, 53-63. 
65. Yang, G.H.; Li, S.; Pestka, J.J. Down-regulation of the endoplasmic reticulum chaperone GRP78/BiP by vomitoxin (Deoxynivalenol). Toxicol. Appl. Pharmacol. 2000, 162, 207-217.

66. Pestka, J.J.; Uzarski, R.L.; Islam, Z. Induction of apoptosis and cytokine production in the Jurkat human $\mathrm{T}$ cells by deoxynivalenol: role of mitogen-activated protein kinases and comparison to other 8-ketotrichothecenes. Toxicology 2005, 206, 207-219.

67. Uzarski, R.L.; Pestka, J.J. Comparative susceptibility of B cells with different lineages to cytotoxicity and apoptosis induction by translational inhibitors. J. Toxicol. Environ. Health A 2003, 66, 2105-2118.

68. Uzarski, R.L.; Islam, Z.; Pestka, J.J. Potentiation of trichothecene-induced leukocyte cytotoxicity and apoptosis by TNF-alpha and Fas activation. Chem. Biol. Interact. 2003, 146, 105-119.

69. Pestka, J.J.; Yan, D.; King, L.E. Flow cytometric analysis of the effects of in-vitro exposure to vomitoxin (deoxynivalenol) on apoptosis in murine T-cells, B-cells and IgA(+)-cells. Food Chem. Toxicol. 1994, 32, 1125-1136.

70. Shi, Y.; Porter, K.; Parameswaran, N.; Bae, H.K.; Pestka, J.J. Role of GRP78/BiP Degradation and ER Stress in Deoxynivalenol-Induced Interleukin-6 Upregulation in the Macrophage. Toxicol. Sci. 2009, 109, 247-255.

71. Parent-Massin, D. Haematotoxicity of trichothecenes. Toxicol. Lett. 2004, 153, 75-81.

72. Bae, H.K.; Shinozuka, J.; Islam, Z.; Pestka, J.J. Satratoxin G interaction with $40 \mathrm{~S}$ and $60 \mathrm{~S}$ ribosomal subunits precedes apoptosis in the macrophage. Toxicol. Appl. Pharmacol. 2009, 237, $137-145$.

73. Ting, J.P.Y.; Duncan, J.A.; Lei, Y. How the Noninflammasome NLRs Function in the Innate Immune System. Science 2010, 327, 286-290.

74. Bae, H.K.; Pestka, J.J. Deoxynivalenol induces p38 interaction with the ribosome in monocytes and macrophages. Toxicol. Sci. 2008, 105, 59-66.

75. Bae, H.; Gray, J.S.; Li, M.; Vines, L.; Kim, J.; Pestka, J.J. Hematopoetic Cell Kinase Associates with the 40S Ribosomal Subunit and Mediates the Ribotoxic Stress Response to Deoxynivalenol in Mononuclear Phagocytes. Toxicol. Sci. 2010, 115, 444-452.

76. Li, M.X.; Pestka, J.J. Comparative induction of $28 \mathrm{~S}$ ribosomal RNA cleavage by ricin and the trichothecenes deoxynivalenol and T-2 toxin in the macrophage. Toxicol. Sci. 2008, 105, 67-78.

77. Yorimitsu, T.; Klionsky, D.J. Eating the endoplasmic reticulum: quality control by autophagy. Trends Cell. Biol. 2007, 17, 279-285.

78. Boddu, J; Cho, S.H., Muehlbauer, G.J. Transcriptome analysis of trichothecene-induced gene expression in barley. Mol. Plant-Microbe Interact. 2007, 20, 1364-1375.

79. Osman, A.M.; Pennings, J.L.A.; Blokland, M.; Peijnenburg, A.; van Loveren, H. Protein expression profiling of mouse thymoma cells upon exposure to the trichothecene deoxynivalenol (DON): Implications for its mechanism of action. J. Immunotoxicol. 2009, doi: 10.3109/15476910903496691.

80. Matsukawa, J.; Matsuzawa, A.; Takeda, K.; Ichijo, H. The ASK1-MAP Kinase Cascades in Mammalian Stress Response. J. Biochem. 2004, 136, 261-265.

81. Iordanov, M.S.; Magun, B.E. Loss of cellular K+ mimics ribotoxic stress. Inhibition of protein synthesis and activation of the stress kinases SEK1/MKK4, stress-activated protein kinase/c-Jun NH2-terminal kinase 1, and p38/HOG1 by palytoxin. J. Biol. Chem. 1998, 273, 3528-3534. 
82. Yamauchi, T.; Keough, R.A., Gonda, T.J.; Ishii, S. Ribosomal stress induces processing of Mybbp1a and its translocation from the nucleolus to the nucleoplasm. Genes Cells 2008, 13, 27-39.

83. Azcona Olivera, J.I.; Ouyang, Y.; Murtha, J.; Chu, F.S.; Pestka, J.J. Induction of cytokine mRNAs in mice after oral exposure to the trichothecene vomitoxin (deoxynivalenol): relationship to toxin distribution and protein synthesis inhibition. Toxicol. Appl. Pharmacol. 1995, 133, 109-120.

84. Pestka, J.J.; Amuzie, C.J. Tissue distribution and proinflammatory cytokine gene expression following acute oral exposure to deoxynivalenol: comparison of weanling and adult mice. Food Chem. Toxicol. 2008, 46, 2826-2831.

85. Amuzie, C.J.; Harkema, J.R.; Pestka, J.J. Tissue distribution and proinflammatory cytokine induction by the trichothecene deoxynivalenol in the mouse: comparison of nasal vs. oral exposure. Toxicology 2008, 248, 39-44.

86. Kinser, S.; Jia, Q.; Li, M.; Laughter, A.; Cornwell, P.; Corton, J.C.; Pestka, J.J. Gene expression profiling in spleens of deoxynivalenol-exposed mice: immediate early genes as primary targets. J. Toxicol. Environ. Health A 2004, 67, 1423-1441.

87. Zhou, H.R.; Yan, D.; Pestka, J.J. Induction of cytokine gene expression in mice after repeated and subchronic oral exposure to vomitoxin (Deoxynivalenol): differential toxin-induced hyporesponsiveness and recovery. Toxicol. Appl. Pharmacol. 1998, 151, 347-358.

88. Zhou, H.R.; Harkema, J.R.; Yan, D.; Pestka, J.J. Amplified proinflammatory cytokine expression and toxicity in mice coexposed to lipopolysaccharide and the trichothecene vomitoxin (deoxynivalenol). J. Toxicol. Environ. Health 1999, 57, 115-136.

89. Zhou, H.R.; Islam, Z.; Pestka, J.J. Kinetics of lipopolysaccharide-induced transcription factor activation/inactivation and relation to proinflammatory gene expression in the murine spleen. Toxicol. Appl. Pharmacol. 2003, 187, 147-161.

90. Clark, I.A. The advent of the cytokine storm. Immunol. Cell. Biol. 2007, 85, 271-273.

91. Roth, R.A.; Harkema, J.R.; Pestka, J.J.; Ganey, P.E. Is exposure to bacterial endotoxin a determinant of susceptibility to intoxication from xenobiotic agents? Toxicol. Appl. Pharmacol. 1997, 147, 300-311.

92. Islam, Z.; King, L.E.; Fraker, P.J.; Pestka, J.J. Differential induction of glucocorticoid-dependent apoptosis in murine lymphoid subpopulations in vivo following coexposure to lipopolysaccharide and vomitoxin (deoxynivalenol). Toxicol. Appl. Pharmacol. 2003, 187, 69-79.

93. Islam, Z.; Moon, Y.S.; Zhou, H.R.; King, L.E.; Fraker, P.J.; Pestka, J.J. Endotoxin potentiation of trichothecene-induced lymphocyte apoptosis is mediated by up-regulation of glucocorticoids. Toxicol. Appl. Pharmacol. 2002, 180, 43-55.

94. Islam, Z.; Pestka, J.J. Role of IL-1 beta in endotoxin potentiation of deoxynivalenol-induced corticosterone response and leukocyte apoptosis in mice. Toxicol. Sci. 2003, 74, 93-102.

95. Islam, Z.; Pestka, J.J. LPS priming potentiates and prolongs proinflammatory cytokine response to the trichothecene deoxynivalenol in the mouse. Toxicol. Appl. Pharmacol. 2005, 211, 53-63.

96. Kolf-Clauw, M.; Castellote, J.; Joly, B.; Bourges-Abella, N.; Raymond-Letron, I.; Pinton, P.; Oswald, I.P. Development of a pig jejunal explant culture for studying the gastrointestinal 
toxicity of the mycotoxin deoxynivalenol: Histopathological analysis. Toxicol. Vitro 2009, 23, 1580-1584.

97. Maresca, M.; Yahi, N.; Younes-Sakr, L.; Boyron, M.; Caporiccio, B.; Fantini, J. Both direct and indirect effects account for the pro-inflammatory activity of enteropathogenic mycotoxins on the human intestinal epithelium: Stimulation of interleukin-8 secretion, potentiation of interleukin-1 beta effect and increase in the transepithelial passage of commensal bacteria. Toxicol. Appl. Pharmacol. 2008, 228, 84-92.

98. Sergent, T.; Parys, M.; Garsou, S.; Pussemier, L.; Schneider, Y.J.; Larondelle, Y. Deoxynivalenol transport across human intestinal Caco-2 cells and its effects on cellular metabolism at realistic intestinal concentrations. Toxicol. Lett. 2006, 164, 167-176.

99. Pinton, P.; Nougayrede, J.P.; Del Rio, J.C.; Moreno, C.; Marin, D.E.; Ferrier, L.; Bracarense, A.P.; Kolf-Clauw, M.; Oswald, I.P. The food contaminant deoxynivalenol decreases intestinal barrier permeability and reduces claudin expression. Toxicol. Appl. Pharmacol. 2009, 237, 41-48.

100. Amuzie, C.J.; Shinozuka, J.; Pestka, J.J. Induction of suppressors of cytokine signaling by the trichothecene deoxynivalenol in the mouse. Toxicol. Sci. 2009, 111, 277-287.

101. Konsman, J.P.; Parnet, P.; Dantzer, R. Cytokine-induced sickness behaviour: mechanisms and implications. Trends Neurosci. 2002, 25, 154-159.

102. Croker, B.A.; Kiu, H.; Nicholson, S.E. SOCS regulation of the JAK/STAT signalling pathway. Semin.Cell. Dev. Biol. 2008, 19, 414-422.

103. Amuzie, C.J.; Pestka, J.J. Suppression of Insulin-Like Growth Factor Acid-Labile Subunit Expression-A Novel Mechanism for Deoxynivalenol-Induced Growth Retardation. Toxicol. Sci. 2010, 113, 412-421.

104. Voss, K.A. A New Perspective on Deoxynivalenol and Growth Suppression. Toxicol. Sci. 2010, 113, 281-283.

105. Pestka, J.J.; Moorman, M.A.; Warner, R.L. Dysregulation of IgA production and IgA nephropathy induced by the trichothecene vomitoxin. Food Chem.Toxicol. 1989, 27, 361-368.

106. Pestka, J.J. Deoxynivalenol-induced IgA production and $\operatorname{IgA}$ nephropathy-aberrant mucosal immune response with systemic repercussions. Toxicol. Lett. 2003, 140-141, 287-295.

107. D'Amico, G. The commonest glomerulonephritis in the world: IgA nephropathy. Q. J. Med. 1987, 64, 709-727.

108. Dong, W.; Pestka, J.J. Persistent dysregulation of IgA production and IgA nephropathy in the B6C3F1 mouse following withdrawal of dietary vomitoxin (deoxynivalenol). Fundam. Appl. Toxicol. 1993, 20, 38-47.

109. Pestka, J.J.; Dong, W.; Warner, R.L.; Rasooly, L.; Bondy, G.S.; Brooks, K.H. Elevated membrane IgA+ and CD4+ ( $\mathrm{T}$ helper) populations in murine Peyer's patch and splenic lymphocytes during dietary administration of the trichothecene vomitoxin (deoxynivalenol). Food Chem. Toxicol. 1990, 28, 409-420.

110. Pestka, J.J.; Dong, W.; Warner, R.L.; Rasooly, L.; Bondy, G.S. Effect of dietary administration of the trichothecene vomitoxin (deoxynivalenol) on IgA and IgG secretion by Peyer's patch and splenic lymphocytes. Food Chem. Toxicol. 1990, 28, 693-699. 
111. Pestka, J.J.; Moorman, M.A.; Warner, R.L. Altered serum immunoglobulin response to model intestinal antigens during dietary exposure to vomitoxin (deoxynivalenol). Toxicol. Lett. 1990, $50,75-84$.

112. Rasooly, L.; Abouzied, M.M.; Brooks, K.H.; Pestka, J.J. Polyspecific and autoreactive IgA secreted by hybridomas derived from Peyer's patches of vomitoxin-fed mice: characterization and possible pathogenic role in IgA nephropathy. Food Chem.Toxicol. 1994, 32, 337-348.

113. Rasooly, L.; Pestka, J.J. Vomitoxin-induced dysregulation of serum IgA, IgM and IgG reactive with gut bacterial and self antigens. Food Chem.Toxicol. 1992, 30, 499-504.

114. Rasooly, L.; Pestka, J.J. Polyclonal autoreactive IgA increase and mesangial deposition during vomitoxin-induced IgA nephropathy in the BALB/c mouse. Food Chem.Toxicol. 1994, 32, 329-336.

115. Zhou, H.R.; Yan, D.; Pestka, J.J. Differential cytokine mRNA expression in mice after oral exposure to the trichothecene vomitoxin (deoxynivalenol): dose response and time course. Toxicol. Appl. Pharmacol. 1997, 144, 294-305.

116. Beagley, K.W.; Elson, C.O. Cells and cytokines in mucosal immunity and inflammation. Gastroenterol. Clin. North Am. 1992, 21, 347-366.

117. Yan, D.; Zhou, H.R.; Brooks, K.H.; Pestka, J.J. Role of macrophages in elevated IgA and IL-6 production by Peyer's patch cultures following acute oral vomitoxin exposure. Toxicol. Appl. Pharmacol. 1998, 148, 261-273.

118. Pestka, J.J.; Zhou, H.R. Interleukin-6-deficient mice refractory to IgA dysregulation but not anorexia induction by vomitoxin (deoxynivalenol) ingestion. Food Chem. Toxicol. 2000, 38, 565-575.

119. Turner, P.C.; Burley, V.J.; Rothwell, J.A.; White, K.L.M.; Cade, J.E.; Wild, C.P. Deoxynivalenol: Rationale for development and application of a urinary biomarker. Food Addit. Contam. 2008, 25, 864-871.

120. Chung Y.J.; Zhou H.R.; Pestka J.J. Up-regulation of macrophage inflammatory protein-2 and complement $3 \mathrm{~A}$ receptor by the trichothecenes deoxynivalenol and satratoxin G. Toxicology 2003, 186, 51-65.

(C) 2010 by the authors; licensee MDPI, Basel, Switzerland. This article is an Open Access article distributed under the terms and conditions of the Creative Commons Attribution license (http://creativecommons.org/licenses/by/3.0/). 